\title{
Open Access Journal of Cardiology
}

\section{Atherosclerosis and Remnant Lipoproteinemia}

\section{Mona Mohammed El Tamalawy* \\ Faculty of pharmacy, Tanta University, Egypt}

*Corresponding author: Mona Mohammed El Tamalawy, Faculty of pharmacy, Tanta University, Egypt, Email: mona.m.eltamalawy@gmail.com

\section{Mini Review \\ Volume 2 Issue 1}

Received Date: December 09, 2017

Published Date: January 05, 2018

\begin{abstract}
Atherosclerosis is the development of complex lipid-laden lesions in the vasculature with the potential to contribute to cardiovascular events including myocardial infarction, stroke and peripheral vascular disease. The normal endothelial cell phenotype is anti-atherogenic, with injury, endothelial cell tight junctions are weakened, allowing egress of lipoproteins into the sub endothelial space, leading to decreased vasodilatory nitric oxide secretion, and finally decrease in flow mediated dilation (FMD). The risk of experiencing a cardiovascular event is 13\% lower per 1\% higher FMD.

Elevated serum LDL is strongly related to the development of atherosclerotic diseases. Epidemiologic studies reveal that hypertriglyceridemia is also associated with atherosclerosis independent of other coronary risk factors. This is a challenge to distinguish high-risk patients from all subjects with hypertriglyceridemia. Atherosclerotic diseases with high triglyceride levels can be found in patients with familial combined hyperlipidemia, diabetes mellitus, and metabolic syndrome, in which triglyceride-rich lipoproteins, especially chylomicron remnants and VLDL remnants, accumulate in the circulating blood and contribute in atherosclerosis. Many researchers have focused on these remnant lipoproteins as atherogenic particles. Remnant lipoprotein (RLP) not only increases in abnormality of lipoprotein metabolism, it is also associated with the progression of atherosclerosis, and coronary artery disease (CAD).
\end{abstract}

Keywords: Remnant lipoprotein; Atherosclerosis; Diabetes mellitus; Coronary Artery Disease

Abbrevations: LPL: Lipoprotein Lipase; TRL: Triglyceride-Rich Lipoprotein; FMD: Flow Mediated Dilation; RLP: Remnant Lipoprotein.

Atherosclerosis is the development of complex lipidladen lesions in the vasculature with the potential to contribute to cardiovascular events including myocardial infarction, stroke and peripheral vascular disease. The blood vessel wall is lined by endothelium which overlies vascular smooth muscle cells while the vessel itself is surrounded by adventitia which includes fibroblasts, adipocytes, and other cells. The classical model of atherosclerosis progression includes injury to the endothelium by circulating factors such as hyperglycemia, hyperlipidemia, inflammatory cytokines, reactive oxygen species, excess flow-mediated stress caused by hypertension, cigarette smoke or environmental toxins. Injured endothelium changes vascular cell phenotype [1].

The normal endothelial cell phenotype is antiatherogenic; healthy endothelial cells have tight junctions preventing entry of lipoproteins into the sub endothelial space; in response to sheer stress, endothelial cells secrete nitric oxide leading to flow-mediated vasodilatation. Furthermore, platelets and monocytes will not adhere to healthy endothelial cells; tissue plasminogen activator, a thrombolytic protein, is secreted by healthy endothelial cells [1]. 


\section{Open Access Journal of Cardiology}

With injury, endothelial cell tight junctions are weakened, allowing egress of lipoproteins into the sub endothelial space. There is decreased function of endothelial nitric oxide synthase leading to decreased vasodilatory nitric oxide secretion, and increased expression of endothelin-1 leading to increased vasoconstriction which means decrease in flow mediated dilation (FMD). Endothelial cells express adhesion molecules which attract and activate platelets and monocyte/ macrophages. In addition, production of plasminogen activator inhibitor type $I$ is increased in injured endothelium. In addition to the specific changes outlined above, there is increased reactive oxygen species, cytokines and growth factors leading to smooth muscle cell proliferation and foam cell formation. Stimulation of adventitial neovascularization have added to the complexity of progression of the atherosclerotic plaque [1].
This chronic or non-resolving inflammation facilitates lipid accumulation in the atheroma, which is a phenotypical hallmark of atherosclerosis. Signs of atherosclerosis may be present throughout the lifetime, fatty streaks - representing the earliest sign of this process - have been observed in fetal aortas, and in children above three years of age [2].

To characterize the degree of atherosclerosis several surrogate markers have been evolved. Two major characteristics of atherosclerosis, endothelial dysfunction and thickening of the vascular wall [1]. Endothelial vasomotor dysfunction has been identified as an early event in the pathogenesis of atherosclerosis, and a surrogate marker of future cardiovascular events. It has been shown previously that lipid-lowering therapies improved endothelial vasomotor dysfunction in patients with remnant lipoproteinemia [3]. FMD is a non-invasive technique for measurement of endothelial vasomotor function as shown in Table 1.

\begin{tabular}{|c|c|c|c|c|}
\hline Study & Population & Follow-up & Conclusions & Limitations \\
\hline Gokce, et al. & $\begin{array}{l}199 \text { patients with peripheral arterial } \\
\text { disease before vascular surgery. }\end{array}$ & 1.2 years & $\begin{array}{c}\text { FMD independently predicts } \\
\text { long-term cardiovascular events. }\end{array}$ & $\begin{array}{c}\text { Only patients with } \\
\text { already established } \\
\text { atherosclerotic disease. }\end{array}$ \\
\hline Huang, et al. & $\begin{array}{l}267 \text { patients with peripheral vascular } \\
\text { disease referred for surgery. }\end{array}$ & 10 months & $\begin{array}{c}\text { FMD predicts cardiovascular } \\
\text { events. }\end{array}$ & \begin{tabular}{|c|} 
Only patients with \\
already established \\
atherosclerotic disease.
\end{tabular} \\
\hline $\begin{array}{c}\text { Brevetti, et } \\
\text { al. }\end{array}$ & $\begin{array}{c}131 \text { patients with peripheral vascular } \\
\text { disease }\end{array}$ & 23 months & $\begin{array}{l}\text { FMD is an independent predictor } \\
\text { of events. }\end{array}$ & $\begin{array}{c}\text { Only patients with } \\
\text { already established } \\
\text { atherosclerotic disease. }\end{array}$ \\
\hline $\begin{array}{l}\text { Chan, } \\
\text { et al. }\end{array}$ & 152 coronary patients & 34 months & $\begin{array}{c}\text { FMD is an independent predictor } \\
\text { of cardiovascular outcome. }\end{array}$ & $\begin{array}{c}\text { Only patients with } \\
\text { oronary artery disease }\end{array}$ \\
\hline $\begin{array}{l}\text { Fathi, } \\
\text { et al. }\end{array}$ & $\begin{array}{l}444 \text { patients deemed at risk of coronary } \\
\text { artery disease }\end{array}$ & 24 months & $\begin{array}{c}\text { FMD is not an independent } \\
\text { predictor of the cardiovascular } \\
\text { outcome. }\end{array}$ & \begin{tabular}{|c|} 
Inclusion of a \\
heterogenic population \\
with several and \\
serious diseases (renal \\
dysfunction, diabetes, \\
advanced coronary \\
diseases). \\
\end{tabular} \\
\hline
\end{tabular}

Table 1: Flow mediated dilation (FMD) as surrogate marker for atherosclerosis.

The association between brachial FMD and CVD risk has been investigated in several prospective studies. Although not conclusive, the majority of these studies showed that FMD is inversely associated with future cardiovascular events. A meta-analysis summarizing the evidence of 14 prospective studies revealed that per $1 \%$ higher FMD, the risk of experiencing a cardiovascular event is $13 \%$ lower [4].
Elevated serum LDL is strongly related to the development of atherosclerotic diseases. Epidemiologic studies reveal that hypertriglyceridemia is also associated with atherosclerosis independent of other coronary risk factors [5]. However, people with slightly elevated triglyceride levels without other metabolic disorders or severe hypertriglyceridemia such as primary chylomicronemia, rarely have CAD. This is a challenge to distinguish high-risk patients from all subjects with 


\section{Open Access Journal of Cardiology}

hypertriglyceridemia. Atherosclerotic diseases with high triglyceride levels can be found in patients with familial combined hyperlipidemia, diabetes mellitus, and metabolic syndrome, in which triglyceride-rich lipoproteins, especially chylomicron remnants and VLDL remnants, accumulate in the circulating blood and contribute in atherosclerosis as seen in (Figure 1) Lipolysis of TRLs induces atherogenesis by generating TRL remnants that transverse the endothelium and lead to foam cell formation in the sub endothelial space. Lipolysis of TRLs also contributes to atherosclerosis and endothelial dysfunction by generating pro-inflammatory, pro-coagulant and pro-oxidant lipid products [6]. Many researchers have focused on these remnant lipoproteins as atherogenic particles [5].

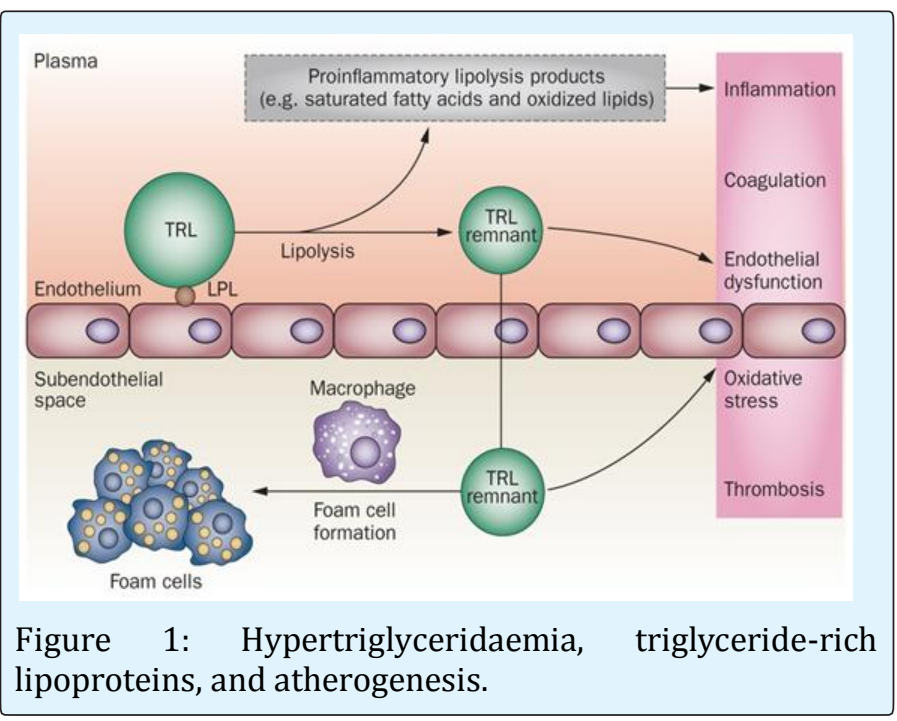

Lipolysis of TRLs induces atherogenesis by generating TRL remnants that transverse the endothelium and lead to foam cell formation in the subendothelial space. Lipolysis of TRLs also contributes to atherosclerosis and endothelial dysfunction by generating pro-inflammatory, pro-coagulant and pro-oxidant lipid products.

\section{RLP-C and Cardiovascular Risk Prediction}

High concentrations of RLP-C in the fasting state were associated with the presence of CAD in dyslipidemia. Kugiyama et al. reported that higher levels of remnant lipoproteins in fasting serum predicted future coronary events in patients with CAD independent of other risk factors. RLP-C and RLP-TG levels were elevated in subjects with not only type 2 diabetes mellitus but also in those with impaired glucose tolerance (IGT), suggesting that patients with IGT have a higher risk for atherosclerosis [5].
Patients at increased risk of CAD frequently exhibit an atherogenic lipoprotein phenotype characterized by elevated plasma levels of both triglyceride- rich lipoproteins, and small, dense LDL and low concentrations of HDL cholesterol [7-9]. Improvement in the predictability of CAD on inclusion of VLDL and IDL cholesterol in the form of non-HDL calculation emphasizes the proatherogenic nature of TRL and their remnant particles. The atherogenic lipoprotein phenotype has been defined by Austin, et al. [10] as the presence of a predominance of small, dense LDL particles, elevated plasma triglyceride levels, and low plasma HDL cholesterol levels in the lipoprotein profile, which is associated with an approximately three-fold increased risk of atherosclerotic disease [9].

The atherogenic lipoprotein phenotype is strongly linked to obesity, insulin resistance, FCH, hypertension, and abnormalities in postprandial lipid metabolism [9].

In the Framingham study, women with diagnosed cardiovascular disease displayed plasma RLP-C and RLPTG levels that were $16 \%$ and $27 \%$ higher than in women without cardiovascular disease [9].

Remnant lipoprotein (RLP) not only increases in abnormality of lipoprotein metabolism, it is also associated with the progression of atherosclerosis, and CAD [3]. Takamitsu Nakamura, et al concluded that elevated RLP-C level was a significant and independent risk factor for impaired FMD and angiographically proven CAD [11]. Treatment with bezafibrate or atorvastatin for four weeks significantly reduced RLP-C levels, with a concomitant improvement in FMD. Therefore, elevated levels of RLP-C are risk factors and predictor for CAD and endothelial vasomotor dysfunction in metabolic syndrome. From this point of view, measurement of RLP$\mathrm{C}$ is useful for assessment of CAD risk, and therapeutic effects in metabolic syndrome [12].

It is important to measure RLP-C level for the assessment of CAD risk in diabetic patients. It is shown in previous literature that high RLP-C level $>0.12 \mathrm{mmol} / \mathrm{L}$ $(7.02 \mathrm{mg} / \mathrm{dL})$ is a significant risk factor for presence of CAD in T2DM $[3,13]$.

A non-fasting remnant cholesterol increase of 1 $\mathrm{mmol} / \mathrm{L}(39 \mathrm{mg} / \mathrm{dL})$ is associated with a 2.8 -fold causal risk for ischemic heart disease, independent of reduced HDL cholesterol. This implies that elevated cholesterol content of TRLs causes ischemic heart disease [14]. 


\section{Open Access Journal of Cardiology}

Multivariate logistic regression analysis showed that high RLP-C levels (> $4.7 \mathrm{mg} / \mathrm{dL}$ ) were a significant risk factor for the presence of CAD, independent of traditional risk factors. Kaplan-Meier analysis demonstrated that higher RLP-C levels in patients with CAD resulted in a significantly higher probability for the development of coronary events. Multivariate Cox hazards analysis showed that high RLP-C levels in patients with CAD were a significant predictor of future coronary events, independent of other risk factors [15]. In another study with a three years follow-up period, the incidence of cardiovascular events in the high RLP-C group (RLP-C > $5.1 \mathrm{mg} / \mathrm{dL}$ ) was higher than that in the low RLP-C group $(\mathrm{RLP}-\mathrm{C} \leq 3.3 \mathrm{mg} / \mathrm{dL})$.

\section{Diabetes \& Cardiovascular Disease}

People with diabetes have a nearly $40 \%$ incidence of cardiovascular disease including CHD, stroke, peripheral vascular disease, and other vascular disease. Prospective longitudinal studies such as Framingham, MRFIT, the Physicians Health Study, and the Nurses' Health Study demonstrated that there was a greater risk of cardiovascular disease for a given blood pressure or cholesterol level in diabetics. The incidence of cardiovascular disease in people with greater than five years of diabetes and in the 55 to 65-years-old age range, was equivalent to cardiovascular risk in persons with previous myocardial infarction. Routine clinical parameters $\mathrm{HbA1c}$, and non-HDL cholesterol predicted CVD in diabetes [16].

When compared to non-diabetic subjects, diabetic patients are at a three (men) to six fold (women) increased risk of suffering a myocardial infarction. Their prognosis is worse and CHD is the main cause of death in this population. Since diabetic subjects without known coronary artery disease have an infraction incidence and a cardiovascular mortality similar to those of non-diabetic subjects with a previous myocardial infarction, all patients with diabetes are considered to be on secondary prevention [17].

Myocardial ischemia due to coronary atherosclerosis commonly occurs without symptoms in patients with diabetes. As a result, multivessel atherosclerosis often is present before ischemic symptoms occur and before treatment is instituted. A delayed recognition of various forms of CHD undoubtedly worsens the prognosis for survival for many diabetic patients [18].

The increase in large VLDL particles is a specific features of dyslipidaemia in insulin resistance and type 2 diabetes which initiates a sequence of events that generates atherogenic remnants. Both LDL and HDL particles show variable compositional changes that are reflected in their functions [19]. Notably apo CIII levels are increased in subjects with T2DM. Together, TRL remnants, small dense LDL and small dense HDL comprise the atherogenic lipid profile, which is also characterized by an increase in apolipoprotein B concentration due to an increased number of apolipoprotein B-containing particles [20]. Importantly, TRLs, including chylomicron VLDL and their remnants, carry a single apolipoprotein B molecule, also like LDL particles. Therefore, the malignant nature of diabetic dyslipidemia is not always revealed by the lipid measures used in clinical practice, as LDL-C may remain within the normal range. It may be better revealed by non-HDL-C. Elevation of TGs or low HDL-C is seen in about half of subjects with T2DM. The abnormal features of the lipid profile precede the onset of T2DM by several years and are common in subjects with central obesity, metabolic syndrome, and T2DM $[21,22]$.

\section{References}

1. Narverud I, Retterstøl K, Iversen PO, Halvorsen B, Ueland T, et al. (2014) Markers of atherosclerotic development in children with familial hypercholesterolemia: A literature review. Atherosclerosis. 235(2): 299-309.

2. Wang JC, Bennett M (2012) Aging and Atherosclerosis Mechanisms, Functional Consequences, and Potential Therapeutics for Cellular Senescence. Circ Res 111(2): 245-259.

3. Nakamura T, Hirano M, Kitta Y, Fujioka D, Saito Y, Kawabata K, et al. (2012) A comparison of the efficacy of combined ezetimibe and statin therapy with doubling of statin dose in patients with remnant lipoproteinemia on previous statin therapy. J Cardiol 60(1): 12-17.

4. Ras RT, Streppel MT, Draijer R, Zock PL (2013) Flowmediated dilation and cardiovascular risk prediction: a systematic review with meta-analysis. Int J Cardiol 168(1): 344-351.

5. Fujioka Y, Ishikawa Y (2009) Remnant lipoproteins as strong key particles to atherogenesis. J Atheroscler Thromb 16(3): 145-154.

6. Watts GF, Ooi EMM, Chan DC (2013) Demystifying the management of hypertriglyceridaemia. Nat Rev Cardiol 10(11): 648-661.

7. Grundy SM (2002) Low-density lipoprotein, nonhigh-density lipoprotein, and apolipoprotein B as 


\section{Open Access Journal of Cardiology}

targets of lipid-lowering therapy. Circulation 106(20): 2526-2529.

8. Frost PH, Havel RJ (1998) Rationale for use of nonhigh-density lipoprotein cholesterol rather than lowdensity lipoprotein cholesterol as a tool for lipoprotein cholesterol screening and assessment of risk and therapy. In: American Journal of Cardiology 81(4A): 26-31.

9. Twickler TB, Dallinga-Thie GM, Cohn JS, Chapman MJ (2004) Elevated Remnant-Like Particle Cholesterol Concentration: A Characteristic Feature of the Atherogenic Lipoprotein Phenotype. Circulation 109(16): 1918-1925.

10. Austin MA, King MC, Vranizan KM, Krauss RM (1990) Atherogenic lipoprotein phenotype. A proposed genetic marker for coronary heart disease risk. Circulation 82(2): 495-506.

11. Nakamura T, Obata JE, Hirano M, Kitta Y, Fujioka D, et al. (2011) Predictive value of remnant lipoprotein for cardiovascular events in patients with coronary artery disease after achievement of LDL-cholesterol goals. Atherosclerosis 218(1): 163-167.

12. Nakamura T, Takano H, Umetani K, Kawabata $\mathrm{K}$, Obata JE, et al. (2005) Remnant lipoproteinemia is a risk factor for endothelial vasomotor dysfunction and coronary artery disease in metabolic syndrome. Atherosclerosis 181(2): 321-327.

13. Yoshida H, Hirowatari Y, Kurosawa H, Manita D, Yanai $\mathrm{H}$, et al. (2012) Estimation of lipoprotein profile in patients with type II diabetes and its relevance to remnant lipoprotein cholesterol levels. Atherosclerosis 222(2): 541-544.

14. Varbo A, Benn M, Frikke-Schmidt R, Tybjærg-Hansen A, Jørgensen AB, et al. (2013) Remnant cholesterol as a causal risk factor for ischemic heart disease. J Am Coll Cardiol 61(4): 427-436.
15. Fukushima H, Sugiyama S, Honda O, Koide S, Nakamura S, et al. (2004) Prognostic value of remnant-like lipoprotein particle levels in patients with coronary artery disease and type II diabetes mellitus. J Am Coll Cardiol 43(12): 2219-2224.

16. Wang CCL, Reusch JEB (2012) Diabetes and cardiovascular disease: Changing the focus from glycemic control to improving long-term survival. Am J Cardiol 110(9): 58-68.

17. Wagner M, Martijnez-Rubio A, Ordonez-Llanos J (2002) Diabetes mellitus and cardiovascular disease. Eur J Intern Med 13(1): 15-30.

18. Grundy SM, Benjamin IJ, Burke GL, Chait A, Eckel RH, et al. (1999) Diabetes and Cardiovascular Disease: A Statement for Healthcare Professionals From the American Heart Association. Circulation 100(10): 1134-1146.

19. Adiels M, Olofsson SO, Taskinen MR, Borén J (2008) Overproduction of very low-density lipoproteins is the hallmark of the dyslipidemia in the metabolic syndrome. Arterioscler Thromb Vasc Biol 28(7): 1225-1236.

20. Taskinen MR, Borén J (2015) New Insights into the Pathophysiology of Dyslipidemia in Type 2 Diabetes 239(2): 483-495.

21. Scott R, Brien R, Fulcher G, Pardy C, D'Emden M, et al. (2009) Effects of Fenofibrate Treatment on Cardiovascular Disease Risk in 9,795 Individuals With Type 2 Diabetes and Various Components of the Metabolic Syndrome. Diabetes Care 32(3): 493-498.

22. Charakida M, Masi S, Lüscher TF, Kastelein JJP, Deanfield JE (2010) Assessment of atherosclerosis: the role of flow-mediated dilatation. Eur Heart J 31(23): 2854-2861. 